\title{
The Effects of Individual Differences on Cued Antisaccade Performance
}

\author{
Alisdair J.G. Taylor \\ University of Sussex
}

\author{
Samuel B. Hutton \\ University of Sussex
}

\begin{abstract}
In the antisaccade task, pre-cueing the location of a correct response has the paradoxical effect of increasing errors. It has been suggested that this effect occurs because participants adopt an "antisaccade task set" and treat the cue as if was a target - directing attention away from the precue and towards the location of the impending target. This hypothesis was tested using a mixed pro / antisaccade task. In addition the effects of individual differences in working memory capacity and schizotypal personality traits on performance were examined. Whilst we observed some modest relationships between these individual differences and antisaccade performance, the strongest predictor of antisaccade error rate was uncued prosaccade latency.
\end{abstract}

Keywords: Prosaccades, Antisaccades, Attention, Working memory, Schizotypy

\section{Introduction}

In the antisaccade task participants are instructed to refrain from looking at a sudden onset target, and direct their gaze instead to its mirror image location. Healthy participants typically fail to suppress an erroneous prosaccade towards the target on around $20-25 \%$ of trials (e.g. Evdokimidis et al 2002). These antisaccade errors are typically followed by a correct saccade to the mirror image location (e.g. Tatler \& Hutton, 2007). Numerous studies have shown that, compared to healthy controls, certain psychiatric and neurological populations make an increased number of such antisaccade errors (see Hutton \& Ettinger 2006; Munoz \& Everling, 2004 for reviews). Increased errors in these patient groups are typically interpreted as reflecting frontally mediated impairments in inhibitory processes.

An often noted (but little studied) aspect of antisaccade performance is that the variability in error rate within healthy controls can be very high. For example, in one large scale study of healthy controls with an average error rate of $23 \%$, the standard deviation was $17 \%$ and the range was $0-100 \%$ (Evdokimidis et al, 2002). Whilst there are many studies describing increased antisaccade error rates in patient populations, there has been comparatively little research into what individual differences might underlie such dramatic variations in healthy control performance.

According to recent accounts, successful antisaccade performance can be considered a function of the extent to which participants are able to adequately activate the task goal (to make an antisaccade) within working memory (Hutton \& Ettinger, 2006; Hutton, Joyce, Barnes \& Kennard, 2002; Nieuwenhuis, Broerse, Nielen \& de Jong, 2004; Reuter \& Kathmann, 2004). As such, one individual difference which may influence antisaccade performance is working memory capacity. This hypothesis was tested directly by Unsworth, Schrock \& Engle (2004) who found that participants with high working memory capacities (as indexed by the operation span task) made fewer errors and had faster correct antisaccade latencies than participants with a low working memory capacity.

Another individual difference that has been found to influence antisaccade performance in healthy controls is schizotypy. There is an extensive literature detailing increased antisaccade errors and correct antisaccade latencies in patients with schizophrenia, and it appears that similar (albeit attenuated) deficits can be observed in 
healthy participants who score highly on measures of schizotypal personality traits (e.g. Gooding, 1999; O’Driscoll, Lenzenwenger \& Holzman, 1998; Larrison, Ferrante, Briand \& Sereno, 2000, Holahan \& O'Driscoll 2005; Ettinger et al 2005). These findings support "dimension" models of psychopathology, in which patients with schizophrenia represent the extreme end of a spectrum of schizotypal personalities (e.g. Claridge. 1997). There is extensive research documenting impaired working memory function in patients with schizophrenia (e.g. Hutton et al, 1998) and some evidence to suggest that deficits in working memory may mediate antisaccade performance in this population (Hutton et al, 2004). There is also evidence that similar cognitive impairments exist, (again to a lesser extent), in healthy participants who score highly on measures of schizotypal personality traits (Park, Holzman \& Lenzenwenger, 1995; Park \& McTigue, 1997; Kopp 2007, although see Lenzenweger \& Gold, 2000). By measuring both working memory capacity and schizotypal personality traits in the same sample, we aimed to determine the extent to which any association between antisaccade performance and schizotypal personality traits observed in healthy participants is mediated by working memory deficits.

In a standard prosaccade task, if the location of the correct response (e.g. the location of the upcoming target) is cued, the latency of the resulting saccade towards the target is reduced (Fischer \& Weber 1998). If, however, the correct location in an antisaccade task is cued (i.e. the mirror image location of the upcoming target), correct latencies are increased, as are the number of errors, (Weber, Durr \& Fischer 1998). This apparently paradoxical finding can be explained within the goal activation model of antisaccade performance outlined above. If participants are successful in activating the correct "antisaccade" task set within working memory, the sudden onset cue may serve as a "go signal" causing attention to be directed to the opposite side, in advance of a saccadic eye movement to that location. If the location to which a correct antisaccade should be directed has been cued, this has the unfortunate effect of directing attention to the opposite side - where the target is about to appear- thus increasing capture errors when the target does appear, and increasing the latencies of saccades made to the correct location.

According to the goal activation hypothesis, within the context of a cued antisaccade task, participants most able to adopt a successful antisaccade task set should be more vulnerable to the effects of cueing than those less able. In other words cueing the correct location should result in a larger decrement in performance in those participants who are in fact best at the task. This prediction was confirmed by Reuter et al (2006) who found that the effect of precuing was weaker in a group of schizophrenic patients, compared to healthy controls. We sought to extend these findings by determining the effects of schizotypy and working memory span on cued antisaccade performance, using a mixed pro / antisaccade task that also allowed us to determine the relationship between pro and antisaccade performance within a single block of trials. We predicted that participants with low working memory span and high scores on a questionnaire measure of schizotypal personality traits would be less susceptible to the cues than high span and low schizotypy individuals.

\section{Methodology}

\section{Participants}

Participants comprised 58 undergraduate students with normal to corrected normal vision from the University of Sussex, of whom 46 were female and 12 male. Ages ranged from $18-31$ years $(\mathrm{M}=24.6, \mathrm{SD}=3.87)$. Participants either received course credit or a small monetary reward. Participants were nave to the purposes of this study. All participants provided written consent, and the experiment was approved by the Life Sciences Ethics Committee at the University of Sussex.

\section{Apparatus}

Eye movements were recorded using an Eyelink II eye tracker (SR-Research Ltd.,Ontario) with a temporal resolution of $2 \mathrm{~ms}$ and a spatial resolution of around 0.25 degs. The stimuli were displayed on a 21 inch CRT monitor with a screen resolution of 1,280 x1,024 pixels and a refresh rate of $100 \mathrm{~Hz}$. Actual screen dimensions were $40 \mathrm{~cm}$ horizontal and $30 \mathrm{~cm}$ vertical. Participants were seated approximately $70 \mathrm{~cm}$ from the screen in an adjustable chair that had been modified to prevent any rotational movement. Each set of trials was preceded by a calibration procedure, during which participants focussed their eye gaze on 9 separate targets in a $3 \times 3$ grid. Only right eye movements were recorded.

\section{Stimuli}

For each trial the display comprised a black background containing two empty marker boxes placed an equal distance $(3.7 \mathrm{~cm}$ from their inner edge to the centre of the screen) on the left and right of a yellow central fixation cross. The marker boxes were $1.5 \mathrm{~cm}$ squared 
and in white. After a brief interval the fixation cross was replaced by a colour circle (subtending $0.8 \mathrm{~cm}$ diameter), which cued the instructions for the present trial. A red circle indicated that an antisaccade was to me made and a green circle indicated that a prosaccade was to be made. The target comprised a red circle $(0.8 \mathrm{~cm}$ diameter $)$ that appeared centered in either of the marker boxes.

\section{Procedures}

The mixed pro / antisaccade task contained 192 trials, 96 of which were prosaccades and 96 of which were antisaccades. After either a short $(500 \mathrm{msec})$ or long (2000 msec) preparation time, the central instruction cue was extinguished and a target appeared in one of the flanking marker boxes. On one third of trials the marker box in which the target subsequently appeared was cued by an increase in its width that lasted for $100 \mathrm{msec}$ before target onset. These trials were designated Cued Same (CS) trials. In another third of trials the flanking box opposite to the one in which the target subsequently appeared was cued (Cued different - CD trials). The final third of trials were uncued (UC trials). In each instruction / cue combination, an equal number of pro and antisaccade trials were administered, with an equal number of long and short instruction delays. The actual order of trials was randomized for each participant. Twelve practice trials were given first.

\section{Measures}

We recorded the number of antisaccade errors (saccades made towards the sudden-onset target) and the latency of correct pro and antisaccade responses. Trials were excluded from analysis if, a) if the eye was not within 40 pixels (approximately 1 degree of visual angle) of the central fixation point at the time of target appearance; b) no saccade was made within the trial duration; c) the primary saccade was obscured by blinks. These criteria resulted in less than $7 \%$ of trials being excluded. The number of errors on antisaccade trials (defined as any saccade made after target onset towards the same hemifield as the target) were counted and expressed as a percentage of the total number of valid antisaccade trials performed. Correct antisaccade latency was measured as the difference in milliseconds between the target onset and the onset of a saccade made to the opposite hemifield (without any intervening erroneous saccade).

\section{Ospan task}

The operation span task (Turner \& Engle 1989) requires participants to solve a series of operation-word problems, whilst trying to remember a set of unrelated words. For each problem, located on the centre of a computer screen, participants are required to read aloud and give a Yes/No response to a simple maths problem and then read an adjacent unrelated word out loud. There are 12 blocks of items - three blocks each of 2,3,4 \& 5 items. After each block, the participant is required to recall as many of the words as possible. For example, a two-item block could be as follows:

- IS $(7 \times 3)-2=4$ ? :CAT,

- IS $(6+2)+1=7$ ? :TREE,

- ???

The first operation-word problem is shown and remains on screen until both the maths part has been solved (the participant answers yes or no) and the unrelated word has been read aloud. The first item is then replaced by the second item and the process is repeated. Finally, the second problem disappears and three question marks appear, which indicate that it is now time for the participant to try to recall as many of the words from that block, in the same order in which they were encountered. Participants are required to write down the recorded words. Blocks consisted of 2-5 operation-word problems and scoring was achieved by totaling the number of correctly recalled words over all blocks.

\section{Schizotypy inventories}

Schizotypal personality traits were measured with the Oxford-Liverpool Inventory of Feelings and Experiences (Mason, Claridge, \& Jackson, 1995). The questionnaire consists of 104 items, divided into four subscales: (i) Unusual experiences, (ii) Cognitive disorganisation, (iii) Introvertive anhedonia, (iv) Impulsive non-conformity. Each item consists of a statement such as "Have you felt that you have special, almost magical powers?" and respondents respond by circling either yes or no. Some items are reverse scored.

The unusual experiences subscale measures "positive" aspects of schizotypal personality such as odd beliefs / magical ideation. Cognitive disorganisation contains items that concern cognitive difficulties such as problems with concentrating and decision making as well as emotional sensitivity and social anxiety. The items in the Introvertive anhedonia subscale measure "negative" aspects of schizotypal personality such as lack of enjoyment in social contact. The Impulsive non-conformity subscale contains items concerned with asocial and impuslive behaviour. 


\section{Results}

\section{Percentage of prosaccade and antisaccade errors}

Percentage of errors for uncued, cued same and cued different trials as a function of preparation time and task are plotted in figure 1 .

Errors were analysed with a three way repeated measures analysis of variance with the factors task: anti vs pro, trial type: UC, CS, CD and preparation time: $500 \mathrm{~ms}$ vs. $2000 \mathrm{~ms}$. There was a highly significant main effect of task (overall more errors were made in the antisaccade task compared to the prosaccade task $\mathrm{F}(1,57)=123$, $\mathrm{p}<$ $.001, \mathrm{r}=.83)$. The main effect of trial type was also significant $(\mathrm{F}(1.4,80)=77.8, \mathrm{p}<.001, \mathrm{r}=.7$, GreenhouseGeisser correction used $\mathrm{e}=.60$ ), with errors being greatest in the CD trials compared to the CS and UC trials. Finally, the main effect of preparation was also significant $(F(1,57)=15.96, r=.47)$ with more errors being made after the shorter preparation time. The task by preparation time interaction was also significant $(\mathrm{F}(1,57)$ $=48.9, \mathrm{p}<.001, \mathrm{r}=.68)$. Main effects analysis revealed that the shorter preparation time resulted in increased errors in the antisaccade task $(F(1,57)=45.7, p<.001 r=$ $.67)$ but slightly decreased errors in the prosaccade task $(\mathrm{F}(1,57)=4.45, \mathrm{p}<.05, \mathrm{r}=.27)$. The task by trial type interaction was significant $(\mathrm{F}(1.4,80)=18.6, \mathrm{p}<.001, \mathrm{r}$ $=.43$, Greenhouse-Geisser correction used $\mathrm{e}=.67$ ) reflecting the fact that errors were increased in the antisaccade task compared to the prosaccade task more in the $\mathrm{UC}$ and CS trials compared to the CD trials. The trial type by time interaction approached significance $(\mathrm{F}(1.7$, 96) $=2.95, \mathrm{p}=.07, \mathrm{r}=.17$, Greenhouse-Geisser correction used $\mathrm{e}=.82$ ) but was qualified by a significant three way interaction between task, trial type and preparation time $(\mathrm{F}(2,114)=9.8, \mathrm{p}<.001, \mathrm{r}=.28)$.

The three way interaction was explored with separate two way: 3 (trial type) by 2 (preparation time) ANOVAs for pro and antisaccade errors. The interaction between trial type and preparation time was significant for the prosaccade trials, reflecting the fact that a longer preparation time resulted in more errors only in the $\mathrm{CD}$ trials $(\mathrm{F}(1.2,68.3)=13.8, \mathrm{p}<.001, \mathrm{r}=41)$ whereas this interaction was not significant for antisaccade trials $(\mathrm{F}(2,114)=$ $1.4, \mathrm{~ns}, \mathrm{r}=.11$ ), reflecting the fact that the shorter preparation time increased errors uniformly across trial types (main effect of preparation time $(\mathrm{F}(1,57)=45.7, \mathrm{p}<.001$, $r=.67)$. The main effects of trial type were significant in both analyses (antisaccades: $\mathrm{F}(1.43,81.25)=9.23, \mathrm{p}=$ $.001, \mathrm{r}=.32$, Greenhouse-Geisser correction used $\mathrm{e}=.71$; prosaccades: $\mathrm{F}(1.4,80)=95.5, \mathrm{p}<.001, \mathrm{r}=.74$, Greenhouse-Geisser correction used $\mathrm{e}=.55$ ).

Paired t-test comparisons revealed that participants made more antisaccade errors in the CD trials compared to the UC trials $(\mathrm{t}(57)=-3.29, \mathrm{p}<.01, \mathrm{r}=.39)$ and the CS trials $(\mathrm{t}(57)=-3.20, \mathrm{p}<.01, \mathrm{r}=.39)$. There was no difference in antisaccade error rate between UC and CS trials, $\mathrm{t}(57)=-.03, \mathrm{p}>.05, \mathrm{r}=.00$. For prosaccade errors, paired t-test comparisons revealed that there were more errors in the $\mathrm{CD}$ trials compared to the $\mathrm{UC}$ trials, $(\mathrm{t}(57)=$ $-8.89, \mathrm{p}<.001, \mathrm{r}=.76)$, and on the CD compared to CS trials, $(\mathrm{t}(57)=-8.98, \mathrm{p}<.001, \mathrm{r}=.77)$. There was no difference with prosaccade error rate between UC and CS trials $\mathrm{t}(57)=.67, \mathrm{~ns}, \mathrm{r}=.09$.

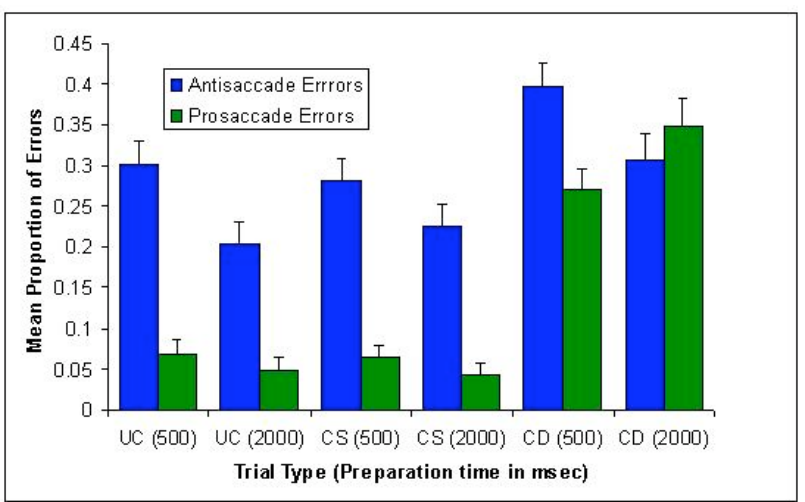

Figure 1. Pro and antisaccade error rates across different trial types.

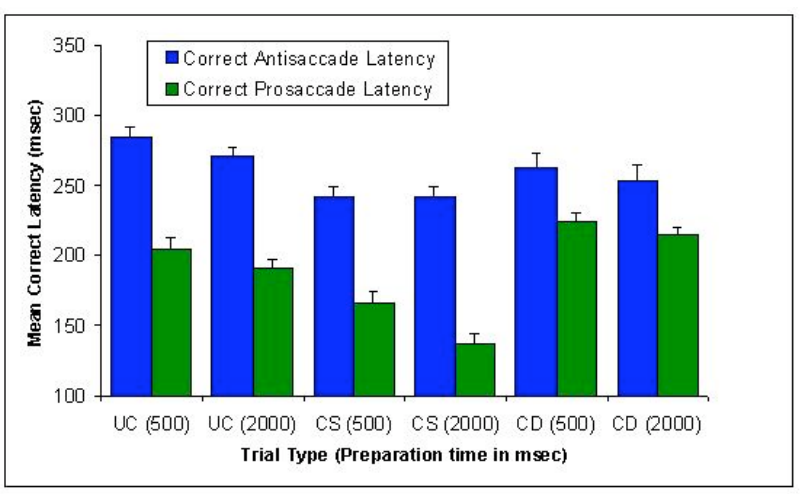

Figure 2. Pro/antisaccade average correct latencies across different trial types. 


\section{Correct latencies}

Correct latencies for uncued, cued same and cued different trials as a function of preparation time and task are plotted in figure 2 . The data was analysed with a three way repeated measures analysis of variance with the factors task: anti vs pro, trial type: UC, CS, CD and preparation time: $500 \mathrm{~ms}$ vs. $2000 \mathrm{~ms}$. As is well established, participants were faster to initiate correct prosaccades compared to correct antisaccades $\mathrm{F}(1,56)=348.31, \mathrm{p}<.001$, $\mathrm{r}=.93$. There was a main effect of trial type, $\mathrm{F}(1.56$, 88.76) $=87.85, \mathrm{p}<.001, \mathrm{r}=.71$, (Greenhouse-Geisser correction used $\mathrm{e}=.79$ ), as latecnies were fastest for CS trials compared to $\mathrm{UC}$ and $\mathrm{CD}$ trials. There was also a main effect of preparation time $(\mathrm{F}(1,56)=20.87, \mathrm{p}<$ $.001, \mathrm{r}=.52$ ), as latencies were fastest overall, when longer preparation time was given. The task by preparation time interaction was also significant, $\mathrm{F}(1,56)=5.62$, $\mathrm{p}=.02, \mathrm{r}=.30$. Main effects analysis revealed that the longer preparation time lead to a greater reduction in latency for prosaccades $\mathrm{F}(1,56)=29.56, \mathrm{p}<.001, \mathrm{r}=.59$. compared to antisaccades $(\mathrm{F}(1,57)=4.84, \mathrm{p}=.03, \mathrm{r}=$ .28). The task by trial type interaction was also significant, $(\mathrm{F}(1.52,85.09)=22.41, \mathrm{p}<.001, \mathrm{r}=.46$ Greenhouse-Geisser correction used $\mathrm{e}=.76$ ), showing that with antisaccades, latencies were faster for CS and $\mathrm{CD}$ compared to $\mathrm{UC}$, whereas only $\mathrm{CS}$ latencies were faster than UC latencies for prosaccades. The trial type by time interaction was not significant, $\mathrm{F}(1.79,100)=.88$, $\mathrm{ns}, \mathrm{r}=.09$ Greenhouse-Geisser correction used $\mathrm{e}=.89$ ). Finally, there was a significant three way interaction between task, trial type and preparation time $(\mathrm{F}(2,112)=$ $5.60, \mathrm{p}<.01, \mathrm{r}=.22$ ).

As with the error data, the three way interaction for latencies was looked at with separate two way: 3 (trial type) x 2 (preparation time) ANOVA's for Pro and antisaccades. There was a significant interaction between trial type and preparation time for prosaccades, $(\mathrm{F}(2,112)$ $=8.60, \mathrm{p}<.001, \mathrm{r}=.27)$, suggesting that a longer preparation time decreased latencies the most for CS trials, but the interaction was non-significant for antisaccades, $(\mathrm{F}(2$, $114)=2.04$, ns, $r=.13$ ). For antisaccades the shorter prepartion time resulted in slower latencies across all trial types (main effect of preparation time; $F(1,57)=4.84$, $p$ $=.03, \mathrm{r}=.28)$. The main effects of trial type were significant in both analyses, (antisaccades: $(F(1.50,85.57)=$ $13.19, \mathrm{p}<.001, \mathrm{r}=.37$, Greenhouse-Geisser correction used $\mathrm{e}=.75$; prosaccades: $\mathrm{F}(1.5,84.23)=162.69, \mathrm{p}<$ $.001, \mathrm{r}=.81$, Greenhouse-Geisser correction used $\mathrm{e}=$ $.75)$.
Paired t-test comparisons revealed that for antisaccades, cueing both the same $(\mathrm{t}(56)=11.78, \mathrm{p}<.001, \mathrm{r}=$ $.71)$ and different sides to the target $(\mathrm{t}(57)=2.96, \mathrm{p}<.01$, $\mathrm{r}=.36$ ), resulted in faster correct latencies, compared to uncued trials. There was also a significant difference between the two cued trials, as correct antisaccades were faster when trials were cued the same side as the target, compared to the different side, $(\mathrm{t}(56)=-2.32, \mathrm{p}=.02, \mathrm{r}=$ $.30)$. For prosaccade latencies, comparisons revealed that there were faster latencies with CS compare to UC trials, $(\mathrm{t}(57)=17.17, \mathrm{p}<.001, \mathrm{r}=.92$. However, participants were significantly slower with CD compared to UC trials, $(\mathrm{t}(57)=-4.97, \mathrm{p}<.001, \mathrm{r}=.55)$. Subsequently, participants were significantly slower with $\mathrm{CD}$ trials compared to $\mathrm{CS},(\mathrm{t}(57)=-13.32, \mathrm{p}<.001, \mathrm{r}=.87)$.

\section{The effects of differences in working memory capacity, on antisaccade performance}

OSPAN failed to correlate with total antisaccade errors (across all trial types), $r=-.20, p=.34$. However, the correlation between Ospan scores and un-cued antisaccade errors, was significant, $r=-.24, p=.03$ (1- tailed). In other words, as Ospan scores increased, uncued antisaccade errors decreased. When one participant with outlying error rate and ospan score was removed from the analysis, the correlation was significant at $\mathrm{r}=-.27, \mathrm{p}=$ .04 (2-tailed). There was no correlation between Ospan scores and correct un-cued antisaccade latency $(r=.02, p$ $=.87)$.

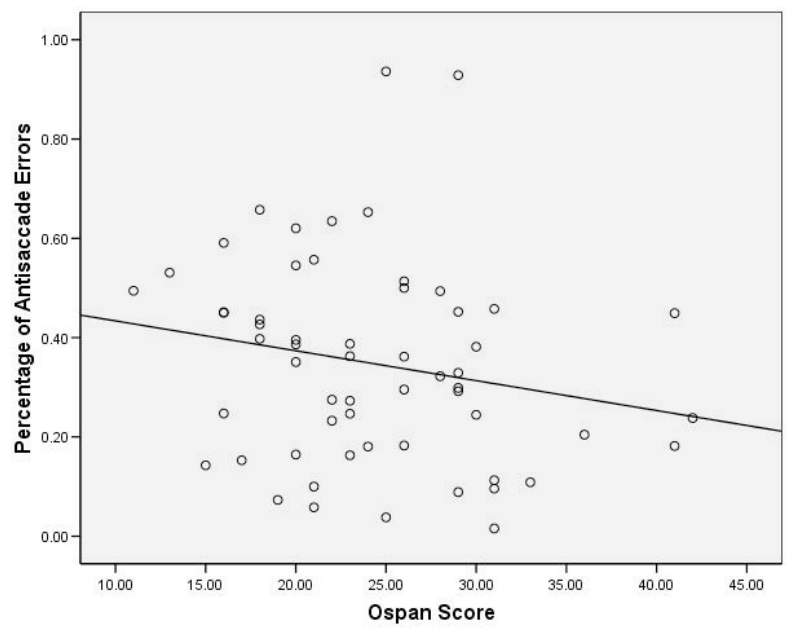

Figure 3. Correlation of Ospan scores and antisaccade errors.

Participants were split on the basis of their ospan scores into two groups, (high vs low working span). A 
mixed $3 \times 2$ ANOVA with trial type (uncued, cued same, cued different) and group (high vs low ospan) as factors revealed a main effect of trial type (Greenhouse-Geisser degrees of freedom correction used, e $=.70,(\mathrm{~F}(1.4$, 78.47) $=9.13, \mathrm{p}=.001, \mathrm{r}=.32)$. There was no effect group $(\mathrm{F}(1,56)=.13, \mathrm{p}=.7, \mathrm{r}=.05)$ nor group by trial type interaction $(\mathrm{F}(2,112)=.7, \mathrm{p}=.5, \mathrm{r}=.08)$. The main effect of trial type reflects the overall increase in errors in the CD condition compared to the UC and CS conditions (see above).

\section{The effects of differences in schizotypal} personality, on antisaccade performance

Total OLIFE scores did not correlate with overall antisaccade errors, $\mathrm{r}=.13, \mathrm{p}=.35$, un-cued antisaccade errors $\mathrm{r}=.05, \mathrm{p}=.7$, or with un-cued correct antisaccade latencies, $r=.01, p=.95$. Scores for the Cognitive Disorganisation and Unusual Experiences subscales of the OLIFE also failed to correlate with any of these antisaccade metrics (all rs $<.21$ ).

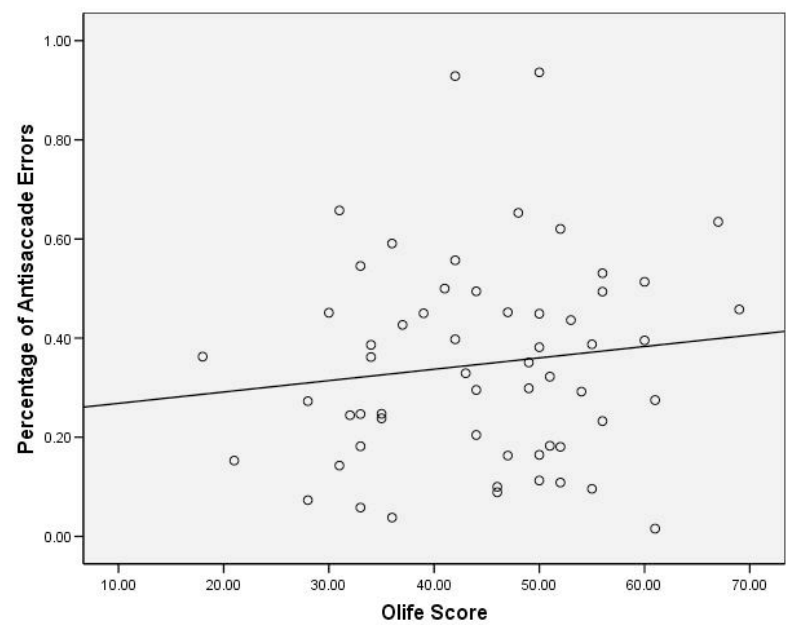

Figure 4. Correlation of Olife scores and antisaccade errors.

Participants were split into high and low groups on the basis of their OLIFE scores. A mixed $3 \times 2$ ANOVA with trial type (uncued, cued same, cued different) and group (high vs low schizotypy) revealed a significant main effect of trial type $F(1.41,79.13)=9.94, p=.001, r$ $=.33$ (Greenhouse-Geisser degrees of freedom correction used, $\mathrm{e}=.71)$. The trial type by group interaction was also significant $\mathrm{F}(2,112)=3.37, \mathrm{p}=.03, \mathrm{r}=.17$. The interaction arises because the low schizotypal group made significantly more errors in the $\mathrm{CD}$ trials compared to the CS trials $(\mathrm{t}(29)=-3.84, \mathrm{p}<.001, \mathrm{r}=.58)$, whereas this difference was not significant for the high schizotypal group $(\mathrm{t}(27)=-.7, \mathrm{p}=.49, \mathrm{r}=.13)$. An ANOVA on correct latencies revealed no main effect of group nor group by trial type interaction (all ps $>.05$ ).

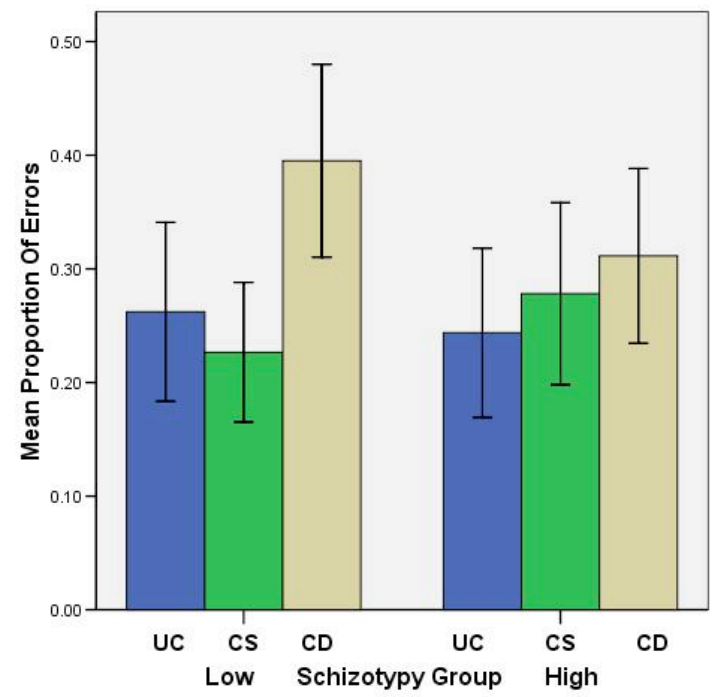

Figure 5. Interaction of trial type and schizotypy.

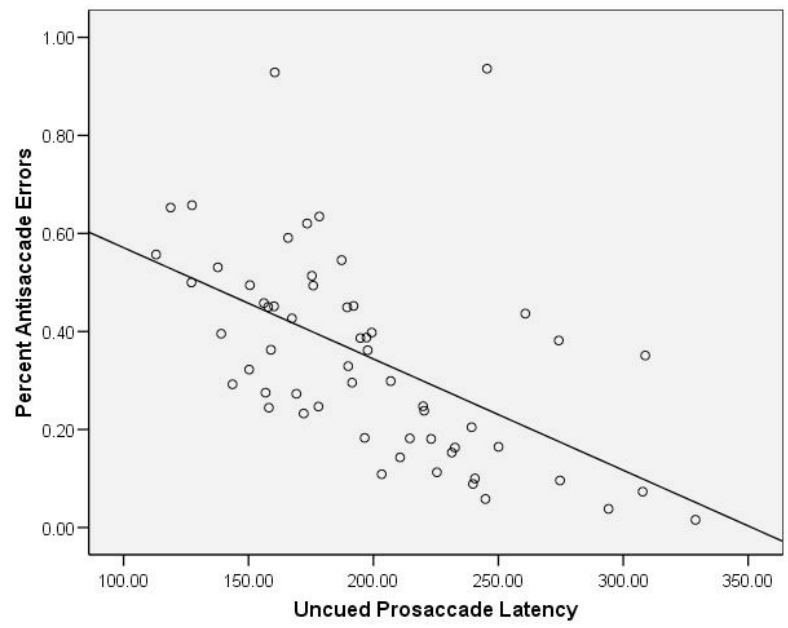

Figure 6. Correlation of pro saccade correct latencies and antisaccade errors. 


\section{The effects of differences in prosaccade performance on antisaccade performance}

Previous research has indicated that antisaccade error rate may be linked to prosaccade latencies (Massen, 2004; Roberts et al, 1994). We found a highly significant correlation between un-cued prosaccade correct latencies and the overall percentage of antisaccade errors (across UC, CS and CD trials), $\mathrm{r}=-.55, \mathrm{p}<.001$ (see figure 4). When the two participants with exceptionally high error rates $(>93 \%)$ were removed, the correlation became even stronger $(r=-.68, p<.001)$. The correlation between uncued prosaccade latency and un-cued errors alone was also significant $(\mathrm{r}=-.6, \mathrm{p}<.001)$.

\section{Discussion}

In this study we explored the effects of a number of individual differences on antisaccade performance. Using a mixed pro and antisaccade task, we found modest relationships between antisaccade error rate and working memory capacity (as measured by OSPAN) and schizotypal personality traits (as measured by OLIFE). The most significant predictor of antisaccade error rate was participant's prosaccade latency. We also replicated and extended previous findings concerning the effects of cueing on antisaccade performance, demonstrating that cueing the location to which a correct antisaccade should be made actually increases error rate.

The small but significant correlation we observed between OSPAN score and un-cued antisaccade error rate, extends the work of Unsworth et al (2004) who observed significant differences in antisaccade error rate between high and low span individuals using the same index of working memory capacity. These findings provide support for models of antisaccade performance that suggest that the ability to sufficiently activate the antisaccade task set within working memory is critical for correct antisaccade performance (Hutton \& Ettinger, 2006; Nieuwenhuis, et al 2004; Roberts, Hager \& Heron 1994; Reuter \& Kathmann, 2004).

We did not however observe the predicted interaction between high and low span and error rate on the CS and $\mathrm{CD}$ trials. We predicted that high span individuals would be most likely to be able to maintain the antisaccade task set "in mind" and therefore show a greater difference in error rate between the CS and CD trials (as they would be more likely than low span individuals to treat the cue as a "go signal" and direct attention to the opposite side, re- sulting in increased attentional capture on CD compared to CS trials). In Unsworth et al's study a large number of participants were screened with the OSPAN task, and antisaccade performance was compared only in those participants who scored in the upper quartile and lower quartile. In our study we did not preselect participants, and whilst the OSPAN score of our high group was very close to that of the high group in the Unsworth et al study (27.3 vs 27.9), our low span group had significantly higher spans (20 vs 6.1). Thus one possibility is that our failure to observe the predicted interation reflects the fact that there were insufficient differences in working memory capacity between our high and low scoring groups. Some support for this suggestion is provided by the fact that both groups made significantly more errors in the CD compared to the CS trials.

The critical interaction between error rates for CS and CD trials was significant when we compared performance in the high vs low schizotypy groups (although the correlations between schizotypy and error rates were not themselves significant). As predicted, high schizotypes demonstrated less of a difference between error rates in the CS and CD trials compared to low schizotypes. This result mirrors the findings of Reuter et al (2006) who compared patients with schizophrenia to healthy controls and found a similar pattern. Our results can be explained if it is assumed that the CD trials increase error rates most in those participants who are comparatively successful at activating the intention to perform an antisaccade, and thus treat the cue as a go signal, and shift attention to the location in which the target subsequently appears. The extent to which this difference reflects differences in working memory capacity between the high and low schizotypy groups is unclear - whilst there was small difference in OSPAN scores in the predicted direction (23 for the low schizotypy group vs 25.7 for the high group), this difference was not significant, and the far larger difference when the groups were split on OSPAN did not produce the expected interaction.

The use of a mixed pro / antisaccade task allowed us to determine the extent to which prosaccade latency determined antisaccade error rate. According to parallel processing, or "race" accounts of antisaccade performance, antisaccade errors occur when activation in the incorrect prosaccade pathway reaches threshold before activation in the correct antisaccade pathway (Cutsuridis et al, 2007; Massen, 2004; Munoz \& Everling, 2004). In support of these models we found a very strong correlation between prosaccade latency and antisaccade error rate. In other words, those participants who tended to 
initiate fast prosaccades towards sudden onset targets were those who made most errors. There are other studies that have observed modest correlations between prosaccade latency and antisaccade error rate (e.g. Roberts et al, 1994), but not all do (e.g. Hutton et al, 1998). These studies used designs in which participants performed prosaccades and antisaccades in separate blocks. It is possible that the strong correlation we observed in the current study reflects the fact that in our mixed design, where the status of each trial was not known until the cue changed to red (antisaccade) or green (prosaccade), activity in the prosaccade pathway remains comparatively high throughout the task, thus increasing its relevance to antisaccade performance.

The overall effects of cueing (irrespective of working memory or schizotypy status) replicated previous findings. Participants made more antisaccade errors when the cue appeared on the opposite side to the target (in other words when the cue actually appeared in the location to which a correct antisaccade should be made). This paradoxical effect replicates Fischer \& Weber's (1996) finding, and suggests that participants treat the cue as if it were the target onset, and direct attention to the opposite side (where the target subsequently appears - thus increasing the likelihood of a capture error). This result is supported by the latency data, which demonstrated that participants take longer to initiate a correct antisaccade if the correct location for that saccade has been cued compared to when the incorrect location is cued.

In conclusion, we found that working memory and schizotypal personality traits had only modest impact on antisaccade performance using a mixed pro / antisaccade task. High schizotypal participants demonstrated a larger increase in antisaccade error rates when the correct location was cued compared to low schizotypal participants, but we did not observe this interaction for high vs low working memory span participants. This latter finding may reflect the relatively modest difference in working memory span between the two groups. Un-cued prosaccade latency emerged as a very strong predictor of overall antisaccade error rate in this task, providing support for parallel processing race models of antisaccade performance (e.g. Massen, 2004). Future research aimed at determining the mechanisms underlying the large variability in antisaccade error rate observed in healthy participants may need to consider as well the potential mechanisms underlying individual differences in prosaccade latencies.

\section{References}

Claridge, G. (1997). Schizotypy: Implications for Illness and Health. New York: Oxford University Press.

Cutsuridis, V., Smyrnis, N., Evdokimidis, I., \& Perantonis, S. (2007). A neural model of decision-making by the superior colicullus in an antisaccade task. Neural Networks, 20, 690-704.

Ettinger U., Kumari, V., Crawford, T. J., Flak, V., Sharma, T., Davis, R. E. \& Corr, P. J. (2005). Saccadic eye movements, schizotypy, and the role of neuroticism. Biological Psychology, 68, 61-78.

Evdokimidis, I., Smyrnis, N., Constantinidis, T. S., Stefanis, N.C., Avramopoulos, D., Paximadis, C., Theleritis, C., Efstratiadis, C., Kastrinakis, G. \& Stefanis, C. N. (2002). The antisaccade task in a sample of 2,006 young men. I. Normal population characteristics. Experimental Brain Research, 147, 45-52.

Fischer, B., Weber, H. (1996). Research note: effects of procues on error rate and reaction times of antisaccades in human subjects. Experimental Brain Research, 109, 507-512.

Fischer B, Weber H. (1998). Effects of pre-cues on voluntary and reflexive saccade generation. I. Anti-cues for pro-saccades. Experimental Brain Research, 120, 403-16.

Holahan, A. V., \& O’Driscoll, G. A. (2005). Antisaccade and smooth pursuit performance in positive- and negative-symptom schizotypy. Schizophrenia Research, 76, 43-54.

Hutton, S. B. (2002). Improved antisaccade performance in schizophrenia with risperidone. $J$ Neurol Neurosurg Psychiatry, 72, 429.

Hutton, S. B. \& Ettinger, U. (2006). The antisaccade task as a research tool in psychopathology: a critical review. Psychophysiology, 43, 302-13.

Hutton, S. B., Puri, B. K., Duncan, L. J., Robbins, T. W., Barnes, T. R., \& Joyce EM. (1998). Executive function in first-episode schizophrenia. Psychological Medicine, 28, 463-473.

Hutton, S.B., Joyce, E. M., Barnes, T.R. \& Kennard, C. (2002). Saccadic distractibility in first-episode schizophrenia. Neuropsychologia, 40, 1729-1736. 
Hutton, S. B., Huddy, V., Barnes, T. R., Robbins, T. W., Crawford, T. J., Kennard, C., \& Joyce, E. M. (2004). The relationship between antisaccades, smooth pursuit, and executive dysfunction in first-episode schizophrenia. Biological Psychiatry, 56, 553-559.

Kopp, B. (2007). Mnemonic intrusions into working memory in psychometrically identified schizotypal individuals. Journal of Behavioural Therapy and Experimental Psychiatry, 38, 56-74.

Larrison, A. L., Ferrante, C. F., Briand, K. A., \& Sereno, A. B. (2000). Schizotypal traits, attention and eye movements. Progressive Neuro- Psychopharmacological Biological Psychiatry, 24, 357- 372.

Lenzenweger, M. F., \& Gold, J. M. (2000). Auditory working memory and verbal recall memory in schizotypy. Schizophrenia Research, 42, 101-110.

Mason, O. Claridge, G. Jackson, M. (1995). New scales for the assessment of schizotypy. Personality and Individual Differences, 18, 7-13.

Massen C. (2004). Parallel programming of exogenous and endogenous components in the antisaccade task. Quarterly Journal of Experimental Psychology Applied, 57, 475-98.

Munoz, D. P. \& Everling, S. (2004). Look away: the antisaccade task and the voluntary control of eye movement. National Review of Neuroscience, 5, 218-28.

Nieuwenhuis, S., Broerse, A., Nielen, M. M., \& de Jong, R. (2004). A goal activation approach to the study of executive function: an application to antisaccade tasks. Brain Cognition, 56, 198-214.

O’Driscoll, G. A., Lenzenweger, M. F., \& Holzman, P. S. (1998). Antisaccades and smooth pursuit eye tracking and schizotypy. Arch. Gen. Psychiatry, 55, 837-843.

Park, S., Holzman, P. S., Lenzenweger, M. F. (1995). Individual differences in spatial working memory in relation to schizotypy. Journal of Abnormal Psychology, 104, 355-363.

Park, S., \& McTigue, K. (1997). Working memory and the syndromes of schizotypal personality. Schizophrenia Research, 26, 213-220.

Reuter, B., \& Kathmann, N. (2004). Using saccade tasks as a tool to analyze executive dysfunctions in schizophrenia. Acta Psychologica (Amst), 115, 255-269.

Reuter, B., Herzog, E., \& Kathmann, N. (2006). Antisaccade performance of schizophrenia patients: evidence of reduced task-set activation and impaired error detection. Journal of Psychiatric Research, 40, 122-130.
Roberts, R. J., Hager, L. D. \& Heron, C. (1994). Prefrontal cognitive processes: working memory and inhibition in the antisaccade task. Journal of Experimental Psychology: General, 123, 374-393.

Rycroft, N., Hutton, S. B. \& Rusted, J. M. (2006). The antisaccade task as an index of sustained goal activation in working memory: modulation by nicotine. Psychopharmacology (Berl), 188, 521-529.

Tatler, B. W., \& Hutton, S. B. (2007). Trial by trial effects in the antisaccade task. Experimental Brain Research, 179, 387-96.

Turner, M. L., \& Engle, R. W. (1989). Is working memory capacity task dependent? Journal of Memory and Language, 28, 127-154.

Unsworth, N., Schrock, J. C., \& Engle, R. W. (2004). Working memory capacity and the antisaccade task: Individual differences in voluntary saccade control. Journal of Experimental Psychology: Learning Memory and Cognition, 30, 1302-1321.

Weber, H., Dürr, N., \& Fischer, B. (1998). Effects of precues on voluntary and reflexive saccade generation II. Pro-cues for antisaccades. Experimental Brain Research, 120, 417-431. 